\title{
Nursing the Patient with Altered Cognitive Function
}

\author{
Jason Cross
}

Cognitive syndromes are common in the older surgical patient. This chapter aims to provide an overview of the causes of altered cognitive function, provide advice on strategies that can be used to identify those at risk and give examples of assessments and interventions to aid diagnosis and treatment. The focus will be on acute confusion or "delirium" but will also comment on how existing cognitive impairment, dementia and depression can impact on patient recovery.

\subsection{Learning Outcomes}

At the end of the chapter, and following further study, the nurse will be able to:

- Identify patients at risk of delirium

- Apply evidence-based tools to assist in the diagnosis and assessment of delirium, depression, cognitive impairment and dementia

- Discuss management strategies and priorities in the patient with delirium, dementia and depression

- Recognise how the ability to give informed consent is impacted by the presence of acute confusional state (delirium) or dementia.

\footnotetext{
J. Cross

POPS Team (Proactive Care of the Older Person Undergoing Surgery), Guys and St Thomas' NHS Foundation Trust, London, UK

e-mail: Jason.Cross@gstt.nhs.uk
} 


\subsection{Assessment of Baseline Health: General Comment}

To recognise a change in an individual's function, there needs to be a robust general assessment to identify and document baseline level of physical and mental health. Comprehensive geriatric assessment (CGA) is an effective way to undertake such assessment, with an evolving literature base detailing improvements in patient and clinical reported outcomes, when utilising CGA methods in preoperative assessment [1]. CGA is considered in more detail in Chap. 4.

\subsection{Delirium}

Delirium is terrifying for the patient (with approximately $50 \%$ recalling the episode) and distressing for family and care workers. All nurses are familiar with the patient who suddenly becomes agitated, aggressive or "not right". Delirium, sometimes called "acute confusional state", is common and can occur after any surgical procedure, with an incidence of up to $60 \%$ after hip fracture. It is categorised by a sudden onset of fluctuating altered consciousness with changes to perception and cognitive function. It is a serious condition that is associated with poor outcomes (see Box 9.1), but it can be prevented and treated with early assessment and intervention [1].

\section{Box 9.1: Consequences of Delirium}

- More hospital-associated complications (pressure injuries, falls)

- Increased stay in hospital or high dependency/critical care in hospital

- Increased incidence of dementia

- More likely to require long-term care/support on discharge

- More likely to die in the short and long term

Managing delirium is challenging, especially when the patient is unable to articulate how they are feeling or is resistant to treatment. Interventions are often only implemented after the patient has developed delirium, with the delirium usually an indicator that the patient is acutely unwell. There is rarely one single predictor of delirium, with it often being a multifactorial combination of long-standing and acute factors. This invariably leads to it being poorly recognised and managed, resulting in increased stress and anxiety for patients, relatives and staff faced with the acutely unwell, rapidly deteriorating, delirious patient.

\subsubsection{Assessment}

Prevention is more effective than a cure, with any intervention needing to begin early. In the surgical setting, this should be done early in the preoperative period. 
Table 9.1 Predisposing and precipitating factors for delirium

\begin{tabular}{l|l}
\hline Predisposing factors & Precipitating factors \\
\hline - Age & - Change in environment \\
- Dementia or cognitive impairment & - Sleep deprivation \\
- Depression & - Loss of sensory aids/clues \\
- History of delirium & - Physical restraints \\
- Severe illness or hip fracture & - Constipation \\
- Polypharmacy & - Urinary retention \\
- Malnutrition/dehydration & - Sepsis \\
- Functional dependency & - Acute illness (e.g. MI) \\
- Sensory impairment & - Untreated pain or excess use of analgesics
\end{tabular}

Assessment can be problematic in emergency/urgent care where time is limited, especially where best practice relies on patients proceeding to surgery as soon as possible. There are "rapid" tools and questions that can assist in identifying those at risk from delirium. Table 9.1 provides a list of predisposing (currently existing) and precipitating (potential causes) for delirium. The prompt recognition of predisposing factors is essential because; (1) many of these factors are modifiable or can be improved and (2) the non-modifiable risk factors raise awareness of the risk of delirium, providing the impetus for interventions.

Cognitive impairment is a predisposing factor for developing delirium and all patients must have cognitive screening on admission to help identify risk. Assessment starts with simple questions such as asking the patient if they have noticed: “... any change in your memory?". This standard screening, used in both hospital and community settings, should then be supported with a more detailed assessment. There are numerous tools and assessments that highlight cognitive decline, but a simple, practical tool to identify those at risk, with one to two more detailed cognitive screening assessment tools to help further assess cognitive deficits and allow onward care planning, is needed.

The "4AT": The 4AT is a brief, easy-to-use, validated tool used to assess for the presence of delirium and identify moderate to severe cognitive impairment [2] with little training needed. It is sometimes preferred to the "abbreviated mental test score" (AMTS) and is free to use and download (www.the4at.com). It can be used for both initial screening and a daily assessment tool to monitor delirium and allows testing of patients with severe drowsiness or agitation. There are four sections with a score for each answer:

1. Alertness: How awake are they? Are they easily woken?

2. AMT4: An abbreviated version of the AMT, asking the patient to recall their location, date of birth and age and to state the current year.

3. Attention: Asked to list the months of the year backwards.

4. Acute change or fluctuating course: Has the patient experienced any hallucinations, paranoia or been acting strangely or "not quite right"?

The more information about baseline cognitive and physical function gathered, the better. A comment made by a family member can provide key information, 
confirming individual risk factors and helping plan individualised interventions. Families may not recognise the subtle changes in cognition that can signify evolving cognitive decline, so questions should be structured to elicit any deficits in function by, for example, asking if there have been any episodes of confusion or any noticeable decline in memory; who does the shopping and manages the household bills; if able to take medications independently; and if placed in the centre of town, on their own, would they be able to make their way home independently? Deficits in these simple tasks indicate potential cognitive decline.

Performing more detailed cognitive screening may not alter the delirium treatment strategy but can help with detailed planning of ongoing care or referral to specialist teams. There are numerous tools; the following two tests can easily be administered by a nurse with appropriate training:

The Montreal Cognitive Assessment (MoCA): (http://www.mocatest.org/) [3] this tests; visuospatial skill, memory recall and attention, takes about 10 min to complete and gives a total score out of 30; a score of less than 26 indicates cognitive impairment.

The Mini Mental State Examination (MMSE): examines different domains with a score out of 30 indicating cognitive decline. This has a larger evidence base and can differentiate between types of dementia [4]. Used for more in-depth assessment of cognition. Losing favour due to copyright: not free to use. Not as good for mild cognitive impairment.

\subsubsection{Identifying Delirium}

Delirium is a medical emergency. Early identification is key to managing the confused patient as well as the acute issue that has been the trigger. Attempts to prevent delirium will not always stop it from developing, so there is a need for sensitive vigilance to any change in patients' behaviour.

The "gold standard" assessment and diagnosis of delirium is the Confusion Assessment Method (CAM), a set of four questions that identify whether a patient is delirious. It is well validated and accurate, with a false-positive rate of $10 \%$. Its use by nurses is often poor [5] and education is needed [6]. The CAM relies on nurses to note subtle changes from baseline behaviour; this is often a comment from a colleague or relative noticing an unexpected change in personality or behaviour. The tool should be used as soon as a change in cognition is suspected and repeated at least daily or if the patient's condition changes. The CAM involves four questions (Table 9.2); delirium is confirmed by a "yes" answer to both questions 1 and 2, with a "yes" to either question 3 or 4 . Some of the components may fluctuate over time, so it is ideal to question others to ensure a full picture.

\subsubsection{Prevention of Delirium}

It is feasible to prevent delirium by modifying risk factors using simple single or multicomponent interventions, e.g. correction of an acute kidney injury (dehydration) by giving intravenous fluids or the prescription of analgesia to manage hip 
Table 9.2 The Confusion Assessment Method (CAM)

1 Is there an acute change in mental status from patient's baseline?

- Is there new confusion, agitation, unusual behaviour, hallucinations, paranoia and/or "just not quite right"?

- Asking a relative or carer can help with this.

What you may hear: "After her hip operation my mother became very confused and aggressive. She kept pulling out her drip and shouting at the nurses. It was a shock as she is usually so polite".

2 Inattention

- Is the patient easily distractible or finding it difficult to follow a conversation?

- To help assess this, you can incorporate the months backwards test (see 4AT).

What you may hear: "I didn't understand what Dad was saying after his operation, one minute he was talking about his knee, and then mentioned being in Germany in the war. Initially I joked with him but he got upset and he was obviously bothered about getting things mixed up".

3 Disorganised thinking

- May not know where they are or think they are somewhere else

- Rambled discussion, jumping from one question to another

- Unable to recall the day or time.

What you may hear: "My wife has mild dementia, but she manages fine at home and we always meet for dinner on Tuesdays. When she was in hospital though, she didn't even recognise me or our daughter; saying we were strangers and there to take her away; it was very upsetting to see".

$4 \quad$ Altered level of consciousness

- Can be aggressive, shouting, anxious or hyper vigilant

- Excessive sleepiness (maybe even unresponsive).

What you may hear: "My uncle became very confused. He was sleepy at times, and agitated and restless at other times. The nurses gave him medication to help control his symptoms".

pain. Interventions targeting the risk factors for delirium include ensuring the patient has their glasses and hearing aids, maintaining day and night routines, promoting sleep and daytime mobilisation and regularly reorientating patients to time and place. All these strategies, when used in a structured protocol, have been shown to significantly reduce the incidence $[7,8]$. It is also essential to communicate with and educate patients and families about the risk of delirium.

\subsubsection{Managing the Delirious Patient}

Using a tool to identify delirium is only helpful if repeated and supported with detailed assessment of the patient's condition. CAM, for example, will provide a diagnosis but does not provide detail about the severity or expected duration of the episode. Once diagnosed, the patient requires close monitoring and rapid intervention to identify the cause and initiate treatment. A delirious patient will lose capacity to make decisions; this is discussed in the dementia section below. The following four actions should happen within the first $4 \mathrm{~h}$ of a diagnosis of delirium:

- Medical review: While this requires medical input, many diagnostic interventions can be initiated by the nurse. Figure 9.2 details the causes of delirium with suggested interventions. Many drugs can cause delirium or make it worse. 
- Falls assessment: Delirious patients are more likely to fall; patients over the age of 65 years having a $30 \%$ risk of falling compared to $10 \%$ of their non-delirious counterparts [9]. A prompt falls risk assessment should be completed with the emphasis on reducing risk (see Chap. 3). A low bed, bed alarms or enhanced observation should be employed to help maintain a safe environment. In the patient who is agitated and wandering, physical restraint is never appropriate; a patient is more likely to settle if allowed to mobilise with support to maintain safety. The use of bed rails is always discouraged as they act as a barrier that can frighten or agitate the patient further, increasing the risk of them climbing over the rails and falling from a greater height; close monitoring is more effective.

- Inform family: Early contact with the patient's family can be the single most effective intervention to assist the healthcare team in the management of the delirious patient. It allows families to feel involved in care, helps reduce stress and provides an opportunity to seek help in managing the delirium. The presence of a relative, friend or carer can be calming, facilitating interventions and relieving the need for close observation by a healthcare team member. This must, though, be done with caution; the presence of a relative with the patient does not reduce the overall risk from delirium, so regular observation and detailed instruction is needed to ensure any change in condition is acted on promptly and appropriately.

- "HELP" interventions: Simple multicomponent interventions, or small actions grouped together in a protocol, reduce the symptoms and duration of delirium. The "Hospital Elder Life Program" (HELP) is a system of patient support that aims to maintain cognitive and physical function during hospitalisation and maximise mobility on discharge, helping with discharge and avoiding hospital readmission [10]. Evidence supports the use of HELP, and the protocol focuses on regular monitoring and intervention. The components include:

- Daily orientation (to time/place)

- Early mobilisation (maintaining function/normal routine, i.e. using toilet)

- Feeding assistance (offering regular drinks/helping with feeding)

- Therapeutic activities (such as board games/playing cards/listening to music)

- A non-pharmacological sleep protocol (maintaining day/night routine; discouraging daytime sleeping)

- Hearing/vision adaptations (ensuring hearing and vision aids).

This strategy is only effective if employed alongside regular, at least daily, medical review. Regular or intentional "rounding" using the above components as a checklist can be helpful, the purpose being facilitating interaction and comfort [11].

\subsubsection{Medication}

A common error in treating delirium is to use antipsychotic medications in excessive doses, give them too late or overuse of benzodiazepines. Sedation in patients with delirium should be avoided and only considered as a last resort if the delirium 
is posing a significant risk to the patient or others. With proactive early assessment and intervention, patients should not need medication, but if they do, the following should be considered (for guidance only; doses and administration should be based on local evidence-based policy):

- Comprehensive patient medical review: identify causes of delirium that could be treated to alleviate agitation

- Delirium may be superimposed on substance withdrawal and additional pharmacological treatments may be indicated

- ECG (electrocardiogram): medications used to treat delirium can cause changes to heart rhythm

- Haloperidol is the first-line treatment in delirium (exceptions below)

- Lorazepam is first line in patients with delirium who also have Parkinson's disease/parkinsonism, Lewy body dementia or seizures, or if the ECG shows changes.

\subsection{Dementia}

Dementia is a collective term for a group of degenerative brain diseases including Alzheimer's disease, vascular dementia, Lewy body dementia and frontotemporal dementia. Worldwide, around 50 million people have dementia and there are nearly 10 million new cases every year [12]. The condition has three stages involving increasing deterioration of memory that impacts on functional and emotional health.

Early stage: Often only recognised in retrospect, when the patient enters the middle or later stages of dementia. The person finds it increasingly difficult to concentrate and becomes more forgetful, exhibiting subtle changes in personality, but often remaining independent functionally, although tasks such as managing finances may become difficult.

Middle stage: Unable to retain short-term memory, able to complete basic personal tasks but with reduced safety awareness and may not be able to leave the home alone, usually requiring support to maintain independent living.

Late stage: Withdrawn and requires full care and support, with limited communication and little to no insight into own condition or environment. Motor deterioration is accelerated and may become bed bound; diet may be reduced due to impaired swallow and increased risk of choking; may not eat at all.

At present there is no cure, although some medications can help with the symptoms. As the age of hospital patients increases, more will present with dementia as either a primary (main cause) or secondary condition (as part of background past medical history). Health practitioners must be aware of the impact of dementia on patients, the associated complications and increased risks to health during treatment. Comprehensive history taking is essential in helping to inform and direct care as discussed in the previous section. 
Cognitive decline impacts on health and decision-making. Understanding the person's individual needs, desires and feelings can be challenging, leading to significant stress for patients, families and staff in acute care situations. Information must be collected from family or friends, or other health practitioners. Dementia "passports" are documents that can be used to describe the patient, their wants, likes and dislikes - illuminating the patient's personality and providing insight into who the patient is so that care can be planned with an individual's values and beliefs maintained [13]; an example is the "This is me" document [13].

\subsubsection{Capacity to Make Decisions}

A central aspect of care for all patients with cognitive difficulties is decisionmaking; be it long term, acute or temporary. Ability to choose what we want and don't want is part of what makes us an individual. To have that ability taken away is distressing and can lead to the values and beliefs of others influencing decisions that may not reflect the individual's own. There are frameworks to help support people with reduced mental capacity in the decision-making process and ensure their best interests are foremost when planning care. In England and Wales, for example, for those over the age of 16, this is the Mental Capacity Act [14], but the following list outlines common principles that help practitioners to best to support patients:

1. Always presume the patient has capacity. They have capacity until proven otherwise.

2. Support people to have capacity through information giving, education and time.

3. People are allowed to make unwise decisions. Not choosing what we perceive as the best course of actions does not indicate a lack of capacity, although may be a sign of reduced understanding.

4. Any treatment must always be in the patient's best interest.

5. If a decision needs to be made without the patient being involved, the least restrictive option should be followed.

Capacity is decision-specific; so when assessing capacity, it is important to know what the question is that requires a decision. For example, it could be a complex decision whether to proceed to surgery or not, involving multiple decisions, or a more simple question regarding taking one medication instead of another. Just because someone may lack capacity regarding one issue, it should not be presumed that this is the same for all issues. If a patient's capacity is in doubt, a two-stage assessment should take place [14]. Box 9.2 is a checklist to make this less daunting.

When an individual who lacks capacity requires treatment, practitioners must ascertain if it is a permanent change in the decision-making process, such as a dementia, or a fluctuating one, such as delirium, drug intoxication or coma. Practitioners must consider what is; (a) in the patient's best interest and (b) the least 


\section{Box 9.2: Two-Stage Assessment for Mental Capacity}

Stage 1

Should be asked of all patients where concern about capacity is an issue:

- Is there a disturbance of consciousness that could cause the patient to lack capacity (YES or NO)? For example, is the patient delirious or has known or newly recognised impaired cognition. If YES—-move to stage 2.

Stage 2

Use a four-point capacity test: "can the patient..."

- Understand the information given relevant to the decision, e.g. information regarding incidence of complication after hip surgery (wound infection, DVT, delirium, success rates etc.)?

- Retain the information given? Can they repeat back the information given? This only needs to be long enough for the decision to be made.

- Weigh up or use the information? Can they discuss the information in context, detailing the pros and cons of the proposed treatment?

- Communicate their decision? Can they say what they want to do?

restrictive option. Practitioners must first ask: can the decision or treatment be delayed to allow time from mental capacity to return? If not, for example, in a timepressured situation such as surgical fixation following fracture, a best interest decision to ascertain the onward course of care is needed.

\subsubsection{Advocacy}

An advocate can only provide opinion and information; the medical or surgical team can note personal preferences and previous decisions made, but this does give the advocate rights to demand or decline treatments that may be in the best interest of the patient. Anyone can advocate for someone as long as they can confidently; (1) state they know the wants and beliefs of the person who they are advocating for and (2) are not in receipt of financial benefit from their relationship (e.g. a paid carer). When patients cannot make a decision for themselves, it is usually a family member or friend who fulfils the advocate role. In a situation where a family/friend is not available in an emergency situation, the surgical or medical team can proceed anyway, using the information they have at hand to make the 'best interest' decision. In non-emergency situations, where capacity is questioned and unlikely to improve, practitioners can seek the help of an independent mental capacity advocate (IMCA), usually appointed by local authorities and who are charged with the gathering and evaluation of information regarding the views of the individual without capacity and make representations on their behalf. An IMCA should always be sought if the following criteria are met: 
- The person is aged 16 or over

- A decision needs to be made about a long-term change in accommodation or serious medical treatment

- The person lacks capacity to make that decision

- There is no independent person, such as a family member or friend, who is "appropriate to consult".

Any best interest "meeting" or discussion must involve as many team members as possible to ensure the decision reached involves aspects that might not be considered by individuals. If all team members cannot be gathered, other forms of communication must be used to ensure all involved are consulted (e.g. via telephone or email) and to ensure all decisions and rationale are documented to provide clarity.

Many countries have human rights legislation that states that all individuals have the right to their liberty being maintained. This can put practitioners in a difficult ethical situation, especially if the patient who lacks capacity resists the treatment being attempted in their best interests, e.g. the "wandering" patient who may try to leave during treatment or the acutely delirious post-operative patient who declines medication. In the UK, for example, the Mental Capacity Act [14] and the Human Rights Act [15] provide guidance on how this can be addressed, providing a framework to legally "deny" the patient without capacity their usual rights to liberty and enforce treatment that is in their best interest. In the UK, an application to apply for a DoLS (Deprivation of Liberty Safeguard) may be instigated through a local government authority, supported by specialist teams. Practitioners should not expect to be able to undertake these comprehensive assessments independently. Once granted, a DoLS can allow restraint and restrictions to keep the patient safe and provide care. Local policies and procedures may differ globally.

\subsection{Depression}

Everyone gets depressed or down from time to time as a normal reaction to life's difficulties, but clinical depression is different; it is a persistent low mood that affects every aspect of a person's life, leading to social isolation, feelings of worthlessness and, in severe cases, to suicide $[16,17]$. The exact cause of clinical depression is not fully understood, although there are recognised risk factors including:

- Personal or family history of depression

- Major life changes, trauma or stress

- Certain physical illnesses and medications.

Depression can occur at any age, but is more likely to develop in adulthood. Simply "being old" does not increase risk of depression, but the accumulation of health issues, along with functional decline and social factors, has been seen to increase the incidence of depression in the older population [18]. Pain, such as chronic back or arthritic pain, is a significant risk factor and, if a diagnosis of 
depression is already present, can increase the severity of the depressive symptoms [19]. Pain is a factor in reduced function for older people and is under-recognised as a cause of depression. "Aches and pains" are often explained away as normal ageing and the individual may not receive the proactive support needed to break the cycle of pain, leading to worsening low mood, loss of function and isolation [20].

Studies recognise that depression impacts negatively on progress after surgery [17]. The symptoms of clinical depression, such as low motivation, perceptions of nonimprovement, sleep disturbance and difficultly with physical rehabilitation, can slow postsurgical recovery [21] with an associated increase in complication rates, with up to $40 \%$ of those with anxiety and depression still suffering the effects after discharge [22].

\subsubsection{Assessment of Depression}

Nurses will often encounter patients with both short- and long-term depression. Clinical assessment and observation should identify existing depression. A comprehensive admission history using alternative sources of information is also essential in highlighting those who may be suffering from depression. In the short term, there may be little that can be done to improve the condition prior to surgery or treatment starting, but assessment can sensitise the team to the risks associated with the depression and allow them to use proactive strategies to help reduce risk. Some of the following will help the practitioner to identify potential for depression [23]:

- History of any mental health disorder

- History of a chronic physical health problem

- Past experience of, and response to, treatments

- Quality of interpersonal relationships

- Living conditions and social isolation

- Family history of mental illness

- History of domestic violence or sexual abuse

- Employment and immigration status.

Nurses can also use an assessment tool to identify those with altered mood, anxiety and depression. There are several tools available, but a simple screening tool will help the practitioner to plan care. Two validated screening tools are recommended by NICE in the UK [24]. These are self-reported questionnaires that give a numerical score; the higher the score, the more likely depression or anxiety will be present:

\section{The Hospital Anxiety and Depression Score (HADS)}

Used for initial diagnosis and to track resolution or progression of anxiety and depression. Validated in many languages and for inpatient and outpatient settings; 14 questions with anxiety and depression questions mixed; these are scored separately with a score of 8 or more in either indicating a positive result; takes 2-5 min to complete [25]. 


\section{The Patient Health Questionnaire 9 (PHQ-9)}

More recently developed tool for monitoring and measuring the severity of depression; sometimes used for screening of depression due to its ease of use; score from questionnaire indicates level of depressive symptoms from no symptoms to severe [26].

As patients complete these themselves, it does not add much time to any assessment. However, these assessments should be viewed with caution as patients can exaggerate symptoms, giving false-positive results. The environment where the test is administered can also skew the results, e.g. a person completing the assessment in a room with other people may lead the individual to answer to fit a social expectation [25]. Some tools are copyrighted with cost to reproduce and print.

\subsubsection{Interventions}

Nurses are well placed to provide the interventions needed to support the patient with depression. Being "at the bedside" provides opportunities to monitor for subtle changes in mood and condition. While the nurse is not expected to be able to perform complex therapeutic techniques, core principles can be integrated into nursing practice. Being caring and compassionate is part of what nurses are, and communication skills and "sixth sense" about potential problems can be an essential component in helping to support patients with anxiety and depression; allowing patients time to talk and express themselves or just letting them be who they are can help build the rapport that can be positive in their recovery.

\subsection{Summary of Main Points for Learning}

- Cognitive disorders are common and precipitants for delirium/acute confusion

- Delirium is a medical emergency with prompt investigation into its cause essential

- Delirium is related to increased in-hospital and post-discharge morbidity (complications) and mortality (death)

- Early assessment with the addition of a simple tool are essential and key to highlighting those at risk

- A structured approach with simple multicomponent interventions can help reduce delirium and its duration; involving family and carers can be of great benefit

- Capacity should always be assessed when altered cognition is present, i.e. delirium or dementia

- Background information is invaluable in understanding the individuals

- A best interest discussion is useful in helping deciding onward care 
- If family or close friend is unable to advocate for a patient with reduced capacity, an independent advocate should be sought

- Any treatment proposed for the patient who lacks capacity should always be the least restrictive option

- Assess early and establish baseline level of needs, both physical and psychological

- Set clear goals for recovery and work with the patient to agree those goals

- Ensure usual psychiatric medications are continued wherever possible

- Consider same staff/team working with the patient to help build rapport

- Reassess depression regularly, allowing time for the patient to discuss their progress, successes and failings.

\subsection{Suggested Further Study}

- Consider how you do, or could, undertake assessment for cognitive status of your patients. Do an internet search for MMSE (Mini Mental State Examination) and compare this with MoCA. Read more about cognitive assessment using the following resource: http://www.mocatest.org/.

- Examine your national and local guidance about capacity, consent and deprivation of liberties and how this is put into practice where you work. How might this impact on your practice? What should you consider doing differently?

- Examine national guidance about caring for patients with dementia in acute hospital settings.

- Look at self-reported assessment tools that are used, or might be used, to identify patients with depression or anxiety in your area. Could a similar tool be integrated into your practice?

\subsection{How to Self-Assess Learning}

- Discuss with your clinical team how you might improve the interventions you use to prevent and manage dementia. Could there be more you could implement in your clinical setting? What plans might you put in place for this? Could this become part of "intentional rounding" in your unit?

- What do you understand about the best way to care for patients with delirium and dementia? Discuss this with colleagues and the carers of patients with dementia. How is this reflected in your current practice?

Acknowledgements The support of Dr. Peter Somerville and Guy's and St Thomas' NHS Foundation Trust delirium and dementia $(\mathrm{DaD})$ team, and permission for the use of the guidance referred to in this chapter, was invaluable. 


\section{References}

1. Partridge JS et al (2017) Randomized clinical trial of comprehensive geriatric assessment and optimization in vascular surgery. Br J Surg 104(6):679-687

2. Bellelli $\mathrm{G}$ et al (2014) Validation of the $4 \mathrm{AT}$, a new instrument for rapid delirium screening: a study in 234 hospitalised older people. Age Ageing 3:496-502

3. Nasreddine $\mathrm{Z}$ et al (2005) The Montreal Cognitive Assessment, MoCA: a brief screening tool for mild cognitive impairment. J Am Geriatr Soc 53(4):695-699

4. Palmqvist $S$ et al (2009) Practical suggestions on how to differentiate dementia with Lewy bodies from Alzheimer's disease with common cognitive tests. Int J Geriatr Psychiatry 24(12):1405-1412

5. Inouye SK et al (2001) Nurses' recognition of delirium and its symptoms: comparison of nurse and researcher ratings. Arch Intern Med 161:2467-2473

6. Wei LA et al (2008) The confusion assessment method: a systematic review of current usage. J Am Geriatr Soc 56:823-830

7. NICE (National Institute for Health and Clinical Excellence) (2010) Delirium: diagnosis, prevention and management, London. https://www.nice.org.uk/guidance/cg103

8. Marcantonio ER et al (1994) A clinical prediction rule for delirium after elective non-cardiac surgery. JAMA 271(2):134-139

9. Pendlebury S et al (2015) Observational, longitudinal study of delirium in consecutive unselected acute medical admissions: age-specific rates and associated factors, mortality and re-admission. BMJ 5:e07808

10. Inouye SK et al (1999) A multicomponent intervention to prevent delirium in hospitalized older patients. N Engl J Med 340(9):669-676

11. Forde-Johnston C (2014) Intentional rounding: a review of the literature. Nurs Stand 28(32):37-34

12. World Health Organization (2017) Dementia. Fact Sheet. http://www.who.int/mediacentre/ factsheets/fs362/en/

13. Alzheimer's Society (2017). This is me-a tool to enable person centred care. https://www. alzheimers.org.uk/download/downloads/id/3423/this_is_me.pdf

14. Mental Capacity Act (2005) London: HMSO

15. Human Rights Act (1998) London: HMSO

16. Richards $\mathrm{C}$ et al (2014) The Oxford handbook of depression and comorbidity. Oxford University Press, New York

17. Rosenberger PH et al (2006) Psychosocial factors and surgical outcomes: an evidence-based literature review. J Am Acad Orthop Surg 14(7):397-405

18. Roberts RE et al (1997) Does growing old increase the risk for depression? Am J Psychiatry 154(10):1384-1390

19. Leite AA et al (2011) Comorbidities in patients with osteoarthritis: frequency and impact on pain and physical function. Rev Bras Reumatol 51:118-123

20. Yohannes AM, Caton S (2010) Management of depression in older people with osteoarthritis: A systematic review. Aging Ment Health 14:637-651

21. Block AR et al (2003) The psychology of spine surgery. American Psychological Association, Washington, DC

22. Tully PJ, Baker RA (2012) Depression, anxiety, and cardiac morbidity outcomes after coronary artery bypass surgery: a contemporary and practical review. J Geriatr Cardiol 9(2):197-208

23. Bowling A (2005) Mode of questionnaire administration can have serious effects on data quality. J Public Health 27(3):281-291

24. National Institute for Health and Clinical Excellence (NICE) (2011) Common mental health problems: identification and pathways to care. https://www.nice.org.uk/guidance/cg123

25. Zigmond AS, Snaith RP (1983) The hospital anxiety and depression scale. Acta Psychiatr Scand 67(6):361-370

26. Hunt $\mathrm{M}$ et al (2003) Self-report bias and underreporting of depression on the BDI-II. J Pers Assess 80(1):26-23 
Open Access This chapter is licensed under the terms of the Creative Commons Attribution 4.0 International License (http://creativecommons.org/licenses/by/4.0/), which permits use, sharing, adaptation, distribution and reproduction in any medium or format, as long as you give appropriate credit to the original author(s) and the source, provide a link to the Creative Commons license and indicate if changes were made.

The images or other third party material in this chapter are included in the chapter's Creative Commons license, unless indicated otherwise in a credit line to the material. If material is not included in the chapter's Creative Commons license and your intended use is not permitted by statutory regulation or exceeds the permitted use, you will need to obtain permission directly from the copyright holder.

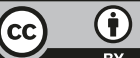

